\title{
POLLY III: A FOUR - VIEW SYSTEM FOR ANALYZING FILM FROM LARGE BUBBLE CHAMBERS
}

F. Beck

Argonne National Laboratory

Argonne, Illinois

August, 1971

(Invited paper at the International Symposium on data hand'ing for Bubble and Spark Chambers, Dubna U.S.S.R., 1971)

thoir contractors, subcontractors, or their employees,

makes any warranty, express or implied, or assumes any

logel liability or responalblitty for the accuracy, com-

pleteness or uxefulness of uny information, epperatus,

product or process disclosed, or represents that its use

would not infringe privately owned rights. 


\section{ABSTRACT}

POLLY is a cathode-ray-tube scanner of high precision, controlled by an on-line computer, and having extensive facilities for help from a human operator. Full use is made of the ability to look at any small part of the picture in great detail, and automatic event-finding and measurement have been performed on a production scale. The system has the advantage that predigitizing, measuring, filtering, and any necessary manual patching can all be performed in one operation.

POLLY III, which is here described in some detail, is the latest of a series of film scanners built at Argonne, and has been designed primarily for use with four-strip $70 \mathrm{~mm}$ film from the Argonne 12-foot hydrogen bubble chamber, but use with film from the projected large chamber at the National Accelerator Laboratory and the big European chambers has been considered.

\section{ACKNOW LEDGEMENT}

The instrument described in this report has been designed and built by a team led by Dr. Bruce Phillips. The mechanical design is the work of Cari Lindenmeyer, and the digital electronics were designed and built by Robert Zieman. Other members of the POLLY hardware team are Gary Thelen, Bob Marema, Harvey Blair and Robert Johnson. Dr. James Bobis, of our electronics division, helped with the design and construction of some of the analog circuitry. The work described here is the culmination of a number of successful hardware and software projects with contributors too numerous to mention here.

$(10,11)$. This work was supported by the U. S. Atomic Energy Commission. 


\section{CONTENTS}

I. Historical Introduction

1.1 The FOLLY Concept

1. 2. POLLY I

1. 3 POLLY II

1.4 Automatic Scanning with POLLY II

1. 5 POILY III

1.6 Other Publications on POLLY

II. Overall Description of POLLY III

2.1 The Measuring Path

2. 2 The Projection Path

2. 3 The CRT Spot

2. 4 Moving the Spot

2. 5 The Slice-Scan

2.6 Detection of Signals

2.7 Operator Controls

2.8 The Reticle

2.9 The Film Platens

2.10 The Indexing Mirror

2.11 The Film Transport

2.12 Th̉e Operator Display

III. Position Control of the Spot on the Precision CR T

3.1 Precision Current Generators

3.2 Ramp Circuits

3. 3 Pincushion Distortion Correction Circuit

IV. Specifications for some Critical Components

4. 1 Precision Lens

4. 2 Deflection Yoke for Precision CR T

4. 3 Precision CR T

4. 4 Fucus Coil

- 4.5 Centering Coil

V. Discriminator Circuit 
VI. Description of the POLLY III Digital Logic

6.1 General

6.2 Digital Control of the Spot on the Precision CRT

6. 3 Information retrieved from the Film

VII. Indexing Motor

VIII. References

IX. APPENDIX: POLLY notes published to July, 1971 


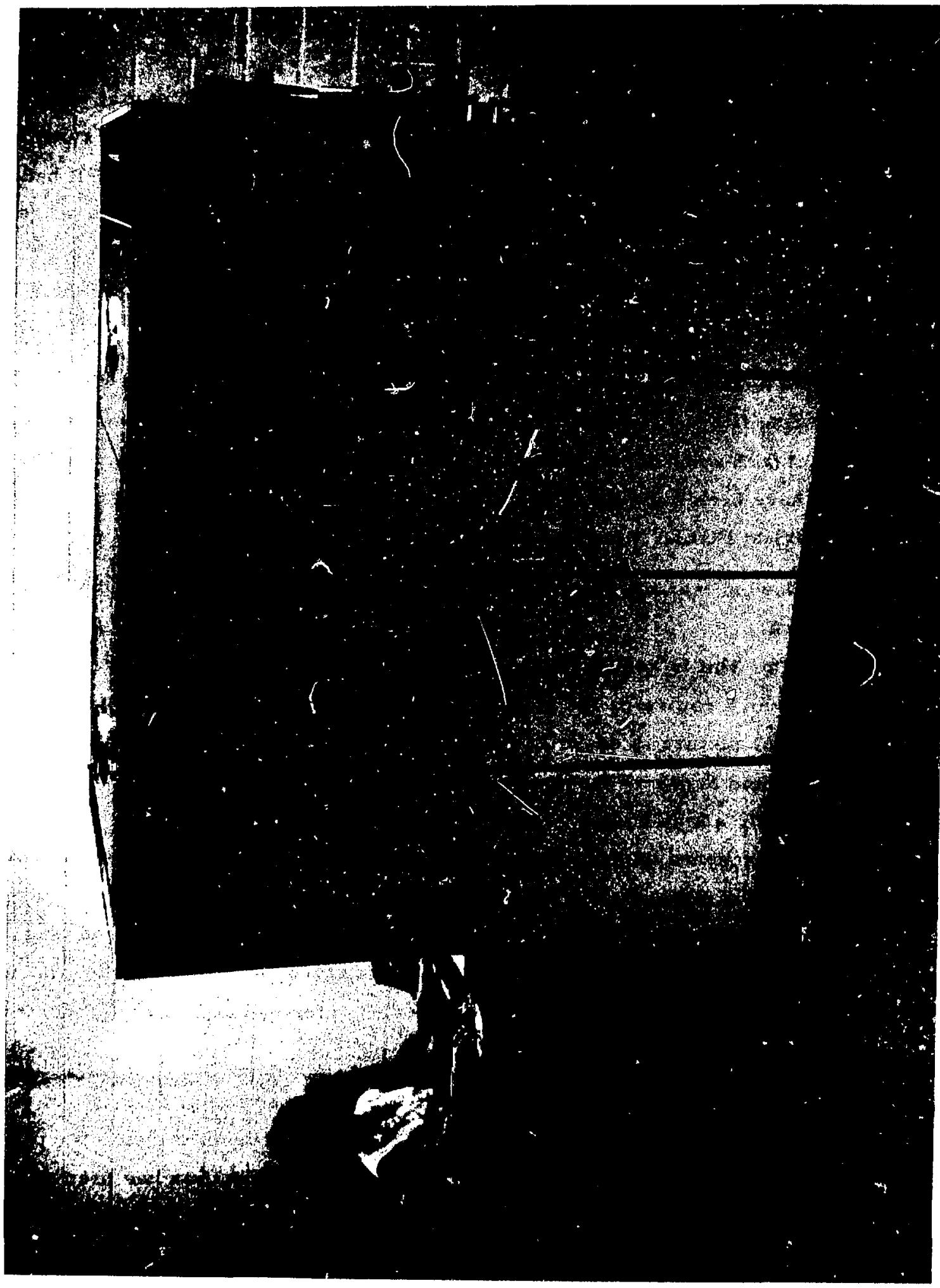


I. Historical Introduction

\subsection{The POLLY Concept}

In 1966, the High Energy Physics Division ai Argonne, deciding that we needed a machine for measuring bubble chamber film at speed, instituted a study of the then available instruments. Since the work of Pless (1) with PEPR had shown the possibilities of CRT devices, and since our Applied Mathematics Division already had some experience of CRT techniques using an instrument called CHLOE ${ }^{(2)}$ on spark-chamber pictures, we decided to support their research efforts on a full-blown bubble chamber measuring project.

The idea as originally planned was to have a computer simultaneously measuring film at several stations, and to arrange for a single operator to interact with whichever station was currentiy in need of help. This device would have been called a poly-measuring machine, and the name was changed (slightly) to POLLY when the original plan was modified.

The following features have been part of the POLLY concept from the beginning:

1. The film is kept in the dark during measuring, except for one spot, which registers the state of the film at that point with a photomultiplier.

2. The picture is swept in lines, the separation of the lines being of the same order as the diameter of the spot.

3. Oniy a small area (about one ten thousandth of the picture in practice) is scanned on any one occasion. Complete computer control is provided of the position, orientation and size of this small area.

4. In view of the known and considerable difficulties of track and event recognition, an operator should be supplied, and the operator should have sufficient information to be well-informed and sufficient control to be helpful.

The first three of these four points were already features of PEPR, and the four th feature has since been added to many of these devices in an effort to increase their flexibility, In POLLY, the operator interaction has been engineered in, as an integral part of the device, and the need for this has influenced many facets of the design, down to the arrangement of the optical paths 
and even the mechanical layout of the cabinet in which it is housed.

Whilst the requirements of 1-4 above have been included in each POLLY machine built, experience taught us a number of valuable lessons. Thus each POLLY built had some new features added as a result of experience, whilst proven principles were retained.

\subsection{POLLY I}

POLLY I was an experimental device. It was put under the control of a small computer, with the idea of building in a track-following algorithm and letting the operator do the rest. Operator intervention was by a track-ball and from a typewriter, and a television picture of the event could be shown by an independent flying-spot scan of the entire picture.

POLLYY I was unexpectedly successful, and minimum-guidance event measuring was shown to be possible. The low resolution of the TV picture proved to be a drawback, and the small computer (PDP-7) was a serious restriction in view of the more ambitious aims which now came into view. However, publicationquality experimental measurements were obtained with POLLY $I,(4,5)$ and while much of the physics community was at that time still unconvinced, we were sure that the most serious problems of stability, linearity and resolution could be solved with a carefully built CRT scanner.

\subsection{POLLY II}

POLLY II was the next instrument in the sequence. A large computer was acquired, which would allow us to put in more elaborate track-following algorithms, and even more significantly to write our programs in FOR TRAN. The advantages of doing this are often underestimated. FOR TRAN programs are easy to modify, several people can work on them at once, and the programmer is not generally loth to throw away a subroutine and re-write it when he has a better idea. All of this is of the essence in a research activity of this kind.

Another very important improvement in POLLY II was the provision of a direct projection of the image being measured, so that the event can be examined in detail by the operator. Since the photomultiplier cannot tolerate high light levels, and projection requires a bright light, operator viewing can oniy take 
place when the machine is not actually measuring. A series of mirrors and shutters is used to convert POLI,Y from a digitizer to a projector under computer control. By providing the operator with an indication of what is happening on the film, we hold his attention so completely that in practice he forgets he is not looking at a projection picture. When the action stops because the program needs help, the indication is reinforced by the appearance on a second screen of a projected image. This arrangement has proved to be so successful that in the design of POLLY III it was deemed unnecessary to pursue the use of colorseparation filters to make a projection available during measuring, even though this is now probably achievable at the state of the art.

The direct-vision screen of POLLY II needed some means for locating features on it, both for the operator to point things out to the computer, and vice versa. An arrangement was devised in which a. servo-controlled marker, driven either from the computer or operator track-ball, was projected on the screen. Finally, the necessity of finding code-letters on a typewriter for communicating with the computer was removed by supplying the operator with a button-board. Thirty-two buttons, with replaceable legends, are available to the operator. A computer-controlled light below each button is conveniently used by the program to infurm the operator which buttons are available to him for his alternative courses of action. This has a small but valuable advantage as a mnemonic aid.

\section{4 Automatic Scanning with POLLY II}

POLLY II considerably surpassed its design goals. It was able to follow the clearly defined, systematically curved and usually well-separated tracks in the Argonne 30 inch bubble chamber so weil that the more elaborate strategy of scanning for beam-tracks could be attempted. Thanks to the availability of greatly increased computer power and the persistence and innovation of Drs. W. Allison and $J$. Loken, the control program was eventually brought to a point where the systematic biases were at an acceptably low levei, and an automatic scanning experiment could be embarked on. The experiment chosen was a $2.3 \mathrm{GeV} / \mathrm{c} \mathrm{p}$ p exposure, in which the comparatively simple scanning rules called for all 2, 4, 6 and 8-prongs inside the fiducial are a to be recorded and measured, stubs to be kept when over $1 / 2 \mathrm{~mm}$ long on the film, and tracks to 
be followed for a $60^{\circ}$ turn in azimuth. Tracks with kinks were followed only up to the kink, and V-zeros were to be ignored at this pass of the film. Some of these restrictions could have been removed by further programming, whilst others, e.g. searching for isolated parts of an event like neutral scatters and V's would require further research. Papers have been published which involve the automatic scanning and measurement of some hundreds of thousands of events with POLLY II (references $6,7,8,9)$.

\subsection{POLLY III}

The main innovation in POLLY III is the provision of four film-transports, any one of which can be used as the measuring station within a fraction of a second. By using an indexing mirror for this purpose we have saved our selves the cost, complication and reliability losses which would have resulted from the use of four separate systems. We have, of course paid in terms of the loss of the capability to switch views at electronic speeds and thus develop a three-dimensional track-following algorithm. An additional drawback of the route we have chosen is that mechanical switching between the views imposes geometrical constraints which are not present in an all-electronic system.

On examination of the inner program loop it was found that the statistical preanalysis of the $r a w$ data from the film occupies a large proporticn of the central processor time in the computer. Since this pre-analysis consiets mainly of finding the centroid of the examined area of the track after rejecting spurious signals, the control circuits of POLLY III were designed to perform these simple discrimination and summing operations automaticaliy, and to pass the pre-digested information to the computer. Thus during trackfollowing the computer rarely needs access to the information from individual scan-lines. If the slice-scan has been correctly placed by the software to fall over the track at approximately the right angle, the hardware will return enough digested information to locate the track element precisely.

\subsection{Other Publications on POLLY}

POLLY is described in detail by a sequence of some thirty POLLY notes, published whilst the various projects were in progress "

(12)

The software has 
been described in exhaustive detail in a two-volume Argonne report (13), and descriptive papers have been published on POLLY I ${ }^{(10)}$, POLLY II ${ }^{(11)}$ and the scanning techniques used with POLLY II (14). The description contained in this report is intended to make it clear what POLLY III is and how it works, and it is hoped that it will be self-explanatory and not require reference to the earlier documentation. 


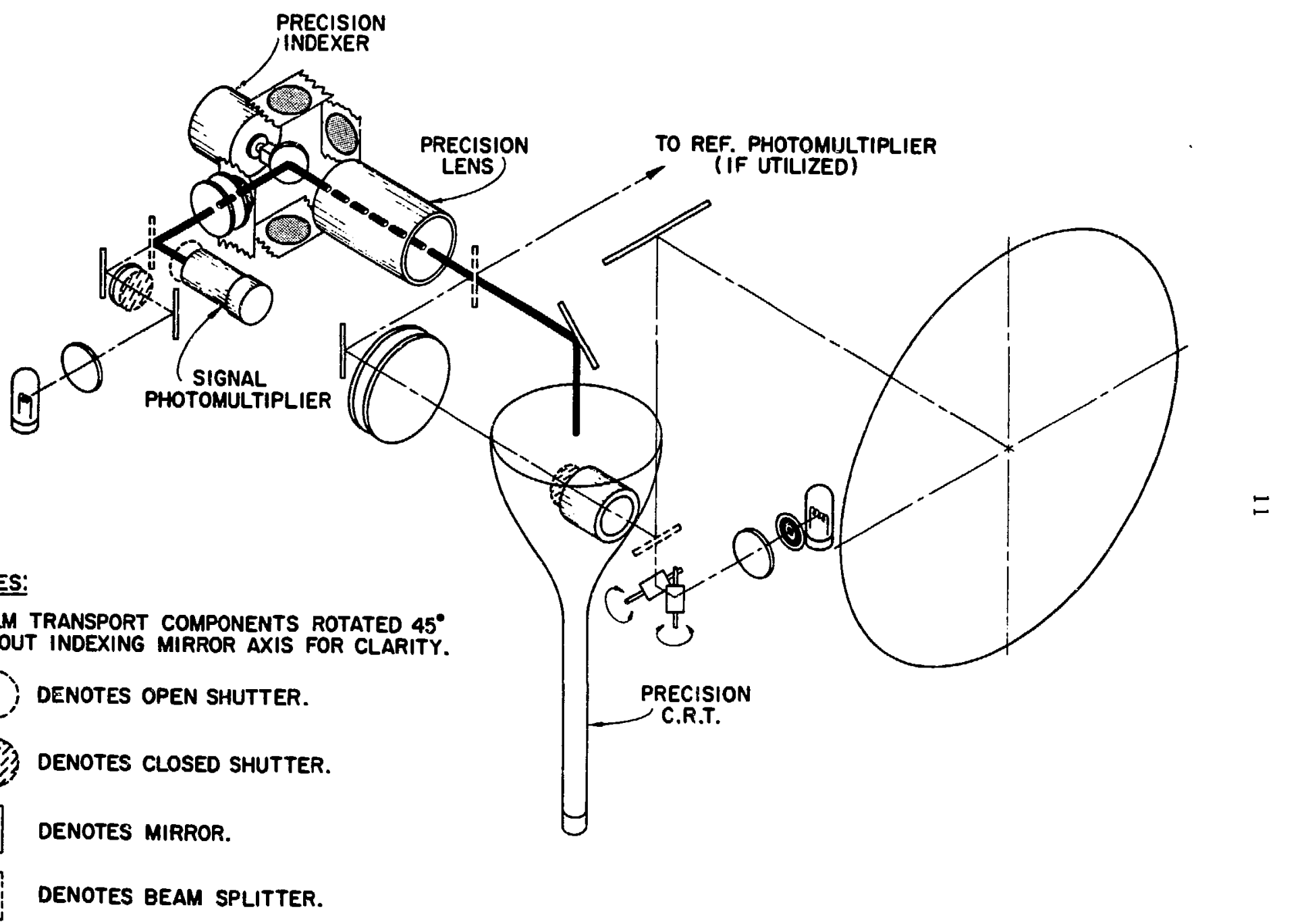

FIG.2 MEASURING PATH 


\section{Overall Descririon of POLLY III}

\subsection{The Mieasuring Path}

POLI. III is a completely self-contained instrument, and is built in to a cabinet 4 it. $\times 7$ ft. $\times 6 \mathrm{ft} .(1 \cdot 2 \times 2 \cdot 1 \times 1 \cdot 8 \mathrm{~m})$ which holds the four film transports and all the measuring and display equipment (Fig. 1) as well as all the power supplies and electronics. Examination of the film by computer is done by using a flying spot from a $9^{\prime \prime}(23 \mathrm{~cm})$ CRT, mounted so that the axis of the gun is vertical. The other components in the measuring train are a front-surface mirror to turn the light path into a horizontal orientation, a specially designed lens giving a half-size image at $32^{\prime \prime}(81 \mathrm{cmi})$, a second front-surface mirror to turn the beam on to one of four film surfaces, and a platen to hold each of the four film-strips flat during measurement. A pellicle introduced on the CRT side of the lens provides an optical path for projecting a direct image of the filin to the operator. The pellicle is dichroic, and passes a little more than half the light in the blue-violet region of the spectrum used by the photomultipliers which are situated behind each film platen. Figure 2 shows the geometrical arrangenient of these elements. All components between the surface of the CR T screen and the film platen are rigidly attached by substantial castings to an optical bench machined out of $2^{\prime \prime}$ thick cast aluminum plate to ensure stability.

POLLY III uses no reference photomultipliers. Experience with the earlier devices showed that the probiems of CR T phosphor granularity are small compared with those arising from low contrast and noise on the film, and therefore the reference signal can be replaced by the use of an autocorrelation prucess. However, the current design would allow of the installation of $a$ reference photomultiplier to sample the light before it passes through the fiim, should it be found desirable in the future.

\subsection{The Projection Path}

So that the operator can obtain a projection image of the film, a projection system is designed into the device. During projection the photomultipliers are protected from stray light by the closing of shutters, and the chosen film is then illuminated by a projection lamp and condenser system. The precision 


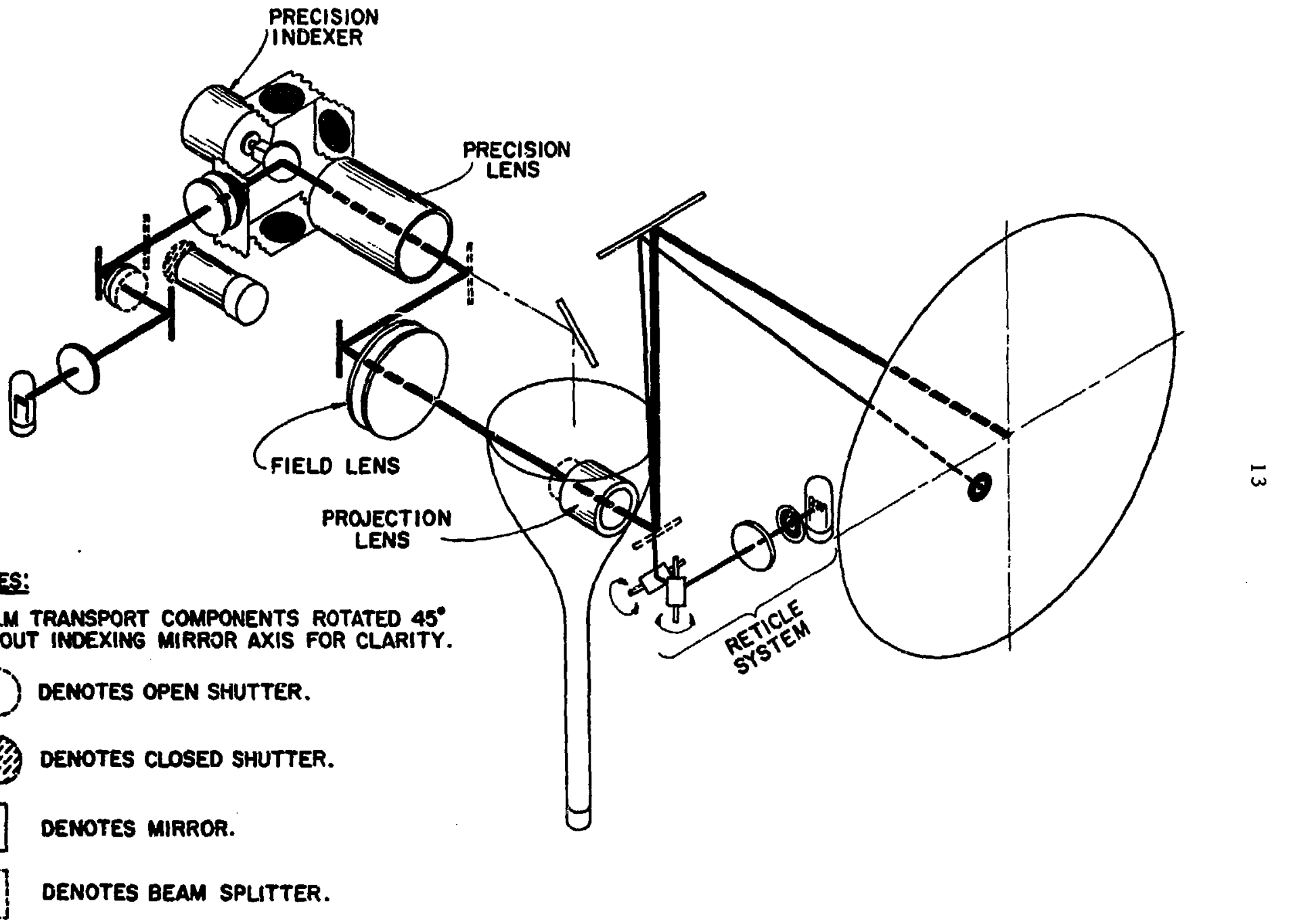

FIG. 3 PROJECTION PATH 
lens, previously used for measuring, throws a blue-violet image of the film on to the CRT, but part of the light is diverted by the pellicle and throws an additional green-yellow image at a second conjugate plane. Into this plane is mounted a field lens to concentrate the light on a projection lens which in turn casts an image on a viewing screen. The path between the field lens and the viewing screen is folded with a pair of mirrors to keep the box to a reasonably short length. Cne of these mirrors is a beam-splitter used for the introduction of a reticle, or pointer image, which is produced by a separate but aligned optical system, and focused on the same screen (Figlire 3).

\section{3 The CRT Spot}

The spot on the CR T projects to a half-brightness diameter of approximately 16 microns. Astigmatism correction and focusing are provided, and extra coils are supplied, with correcting currents to hold the focus and as tigmatism correction under control away from the center of the screen.

\section{4 Moving the Spot}

The spot can be steered to any of $32768 \times 32768$ precisely determined positions by means of very precise, stable D-A current generators controlled from digital counters. While 32768 positions implies a 15 -bit D-A converter and such a converter is actually used, the required precision is actually an order lower than this, and only 12 bits are selected from the computer, giving 4096 $x 4096$ major mesh points. The other three bits are used for steering the spot over the exact pattern required by the automatic hardware, and constitute 32768 $\times 32768$ minor mesh points.

Thus starting with a $65.536 \mathrm{~mm}$ image on the film (it is actually a little larger than this, but $65.536 \mathrm{~mm}$ is a convenient figure) we can have a major meshpoint every $16 \mathrm{microns}$ in $X$ and $Y$, and a minor mesh-point every 2 microns.

When sweeping, the spot is controlled by an analog ramp circuit, linear with respect to time in $X$ and $Y$. The current from this ramp circuit, plus the analog-computed correction for pincushion distortion, is added to the D-A output before application to the deflection coils. Thus the spotstarts at a chosen major or minor deflection point and proceeds across the face of the tube at an angle 
determined by the ratio of the $X$ and $Y$ ramp currents.

\section{5 The Slice-Scan}

The actual path of the spot is the sc-called slice-scan. A slice-scan consists of a number of parallel sweeps separated by about one spot diameter (Figure 4). The starting points of all the sweeps are collinear and on a straight line perpendicular to each sweep, as are the end points. The angle which the start points (and end points) make with the $X=0$ direction is called the oriantation of the slice-scan, and in POLLY III only 64 orientations are possible. These orientations are not uniformly distributed in angle, but are obtained by having the start point of each line eight, 2 micron steps away from the $s$ tart point of the previous line in one direction, and some integral number of: steps between 0 and 7 in the or thogonal direction.

By arranging for the ramp circuit which moves the spot in one direction to deflect it at 4 microns per microsecond, and that which moves it in the or thogonal direction to deflect it at a suitable fraction $10 / 8,1 / 8,2 / 8$. . $7 / 8$, $8 / 8)$ of 4 microns per microsecond, the spot is made to travel across the picture in a direction perpendicular to the orientation.

At the same time as the ramp circuit is moving the spot across the picture, a clock counts microseconds. Thus the time of any event encountered by the spot can be correlated with the instantaneous position of the spot, by aciding a suitably weighted clock-time to the already known coordinates of the startiug point. Figure 5 will hopefully make this clear.

In use, the POLLY hardware is told the coordinates of the major deflection point which is to be a corner of the slice-scan, the orientation and the length of each sweep, and then asked to execute a certain number of sweeps. The digital logic, which is described in section VI, then sets the ramp attenuation factors and gives the signal for the ramp circuits to draw the first line. The spot is then blanked and steered to the minor deflection point at which the second line is to start, when bright-up is applied and another scan-line is drawn. This process continues until the entire slice-scan is complete. 


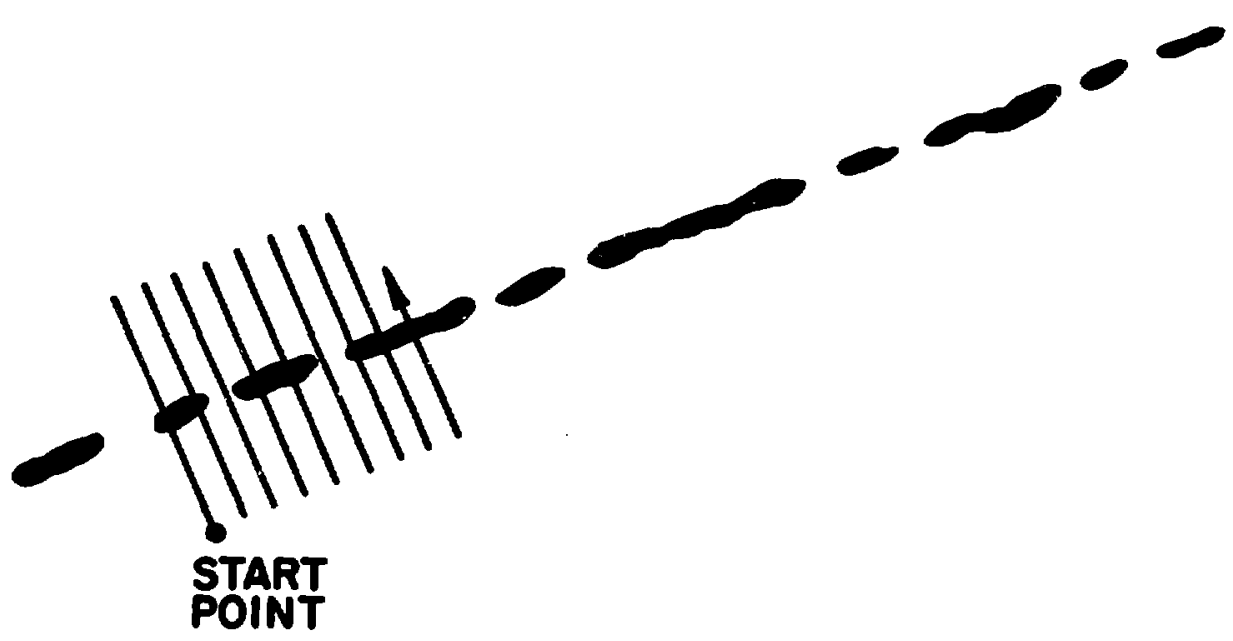

FIG. 4 POLLY SLICE SCAN 


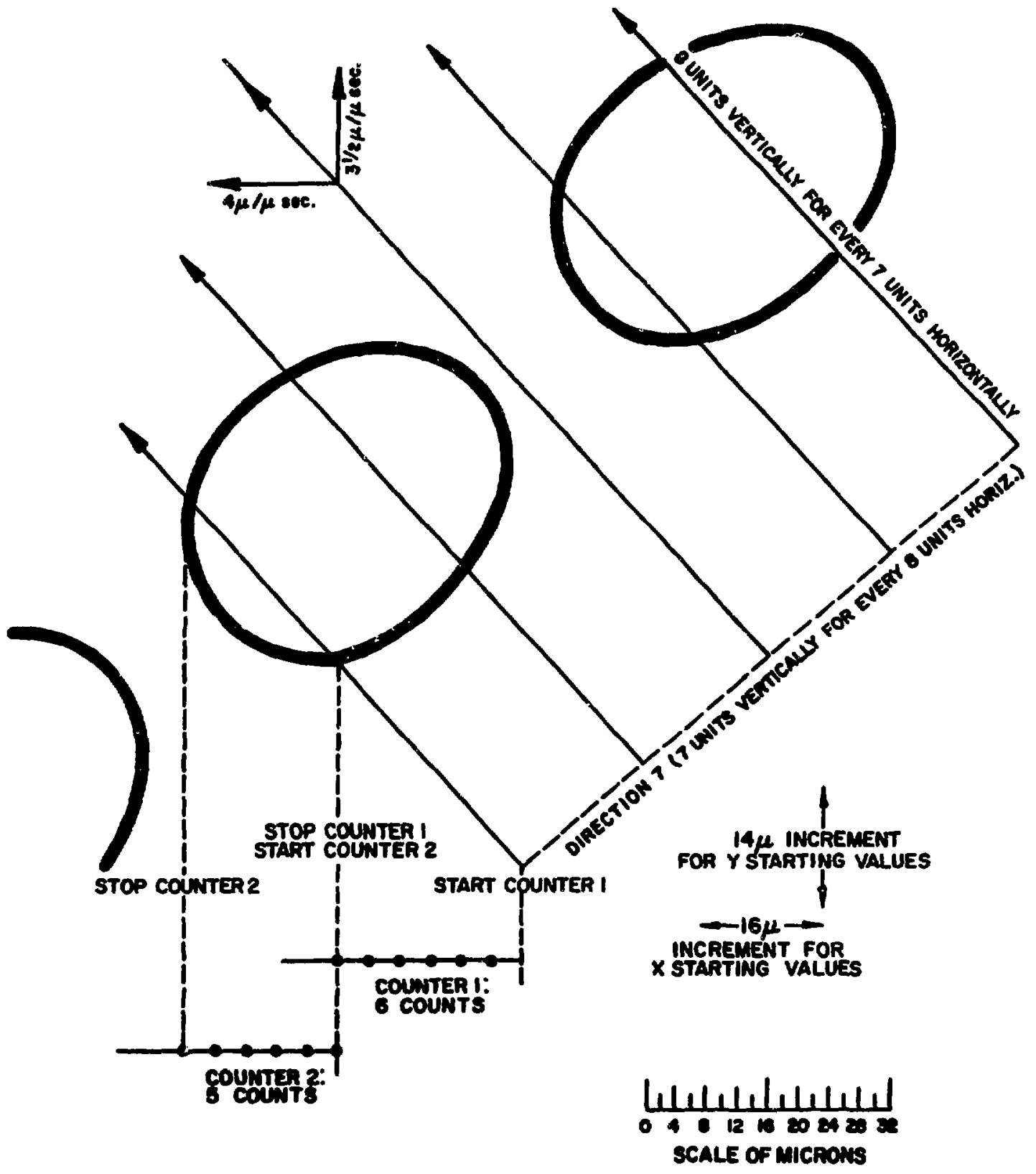

FIG.5

POLly III Slice scan structure 


\subsection{Detection of Signals}

All the while the spot is moving across the film, the photomultiplier behind the film is recording signals. The output of the photomultiplier is fed into a discriminator circuit, which is given the task of deciding whether the signal it receives comes from film background or from a track or fiducial mark. Three computer-loaded registers control parameters used in making this decision. One, the "discriminator level" sets the noise-level, and the other two set the lower and upper width limits betweer which a signal will be accepted as coming from a track. The discriminator is described in Section $V$.

At each scan-line the discriminator is reset. If one and only one good track signal is detected, above the designated noise and of a sufficient but not excessive width, the line is considered to have given a good track crossing. The clock-time from the start of the line to the beginning of the track is sent to the computer, as is the clock time across the width of the line. Registers carrying sums useful for finding the centroid are suitably incremented.

If no track element is found on a particular scan-line, or if a second good crossing is found after one has already been recorded, the line is designated a "bad track crossing" and other sums are incremented. In the case of a multiple hit a flag is transmitted to the computer with the edge and width information.

\subsection{Operator Controls}

The operator controls consist of a track-ball, a knob and a set of 32 buttons. Encoders on the ball send out a stream of pulses to an X-register as it is turned, together with a signal which causes incrementation when $X$ increases and decrementation when $X$ decreases. There is of course also a $Y$ register and the ball also sends out pulses and signals for $Y$. A knob and another register are also supplied for specifying angles. The $X, Y$ and angle registers can be loaded or read from the computer.

The 32 buttons on the operator's console are connected to a register of 32 bits which can be read from the computer. If any button is pushed, the bit corresponding to that button is set to 1 , and all others are set to zero. There is a light under each button, and these 32 lights can be controlled by sending a 
32 -bit word from the computer to a 32-bit register. Each 1 causes the corresponding light to be illuminated, and each 0 causes the corresponding light to be extinguished.

\subsection{The Reticle}

The reticle or pointer is produced by a small, brightly illuminated hole and concentric circle on a silvered transparent surface with a condenser lens to collimate the light. The light is deflected in the $\mathrm{X}$ and $\mathrm{Y}$ directions by a pair of mutually perpendicular front-surface mirrors which can rotate under the control of servo-motors. These motors are driven from the D-A converters which are used as the basis of pincushion corrections in the precision circiats. (See Figure 8). To avoid the reticle attempting to follow the scanning process the servo-motors are electrically isolated to hold the reticle ia place when measuring is in progress.

\subsection{The Film Platens}

The film platens are rigidly attached to a casting holding the indexing mirror. Each platen has a glass plate with grooves which are evacuated during meas uring, clamping the film into firm contact with the glass. The film transports are attached to the platens only by loose loops of film, and their position and alignment are not critical to the measurement process. During film movement the film is blown clear of the platen to avoid scratching.

\subsection{The Indexing Mirror}

The indexing mirror which aligns the optical path with that one of the four views which is currently being measured has to have great stability and repeatability. A commercially available stepping-motor was chosen, which has these features, as well as the desirable one that it is designed to wear symmetrically and thus remain stable and repeatable. Bench tests have shown the repeatability of the indexing motor to be within less than 5 seconds of arc, and this is sufficient to ensure return of the picture within one micron after indexing. Section VII describes the indexing motor. 


\section{11 The Film Transport}

The film transports on POLLY III are almost unmodified versions of ones which have been used successfully on previous POLLIES and on Argonne's $12 \mathrm{ft}$. measuring table. Automatic control of the film is available by means of stepping motors driven from electronic counters. Ramp circuits control acceleration in a linear fashion up to a speed of 200 inches per second, and a speed schedule is initiated in a similar fashion a fixed distance from the end of the desired movement. The reels are driven by motors actuated by a servomechanism using loops of film in the conventional way. The mechanical layout of the four transports conveniently corresponds to that of the chambers in the 12 ft. bubble chamber at Argonne.

\subsection{The Operator Display}

The operator display of POLLY III is a commercially available one. It is a high-quality CR T display with a vector generator, character generator, point generator and the capability of displaying sequences of characters and vectors without specifying the position each time. The display has no buffer memory, and is refreshed by a channel from a dedicated area of the computer memory. Apart from its physical position in the box, the display is in no way connected with the rest of POLLY, and simply shares the same operator and computer. 


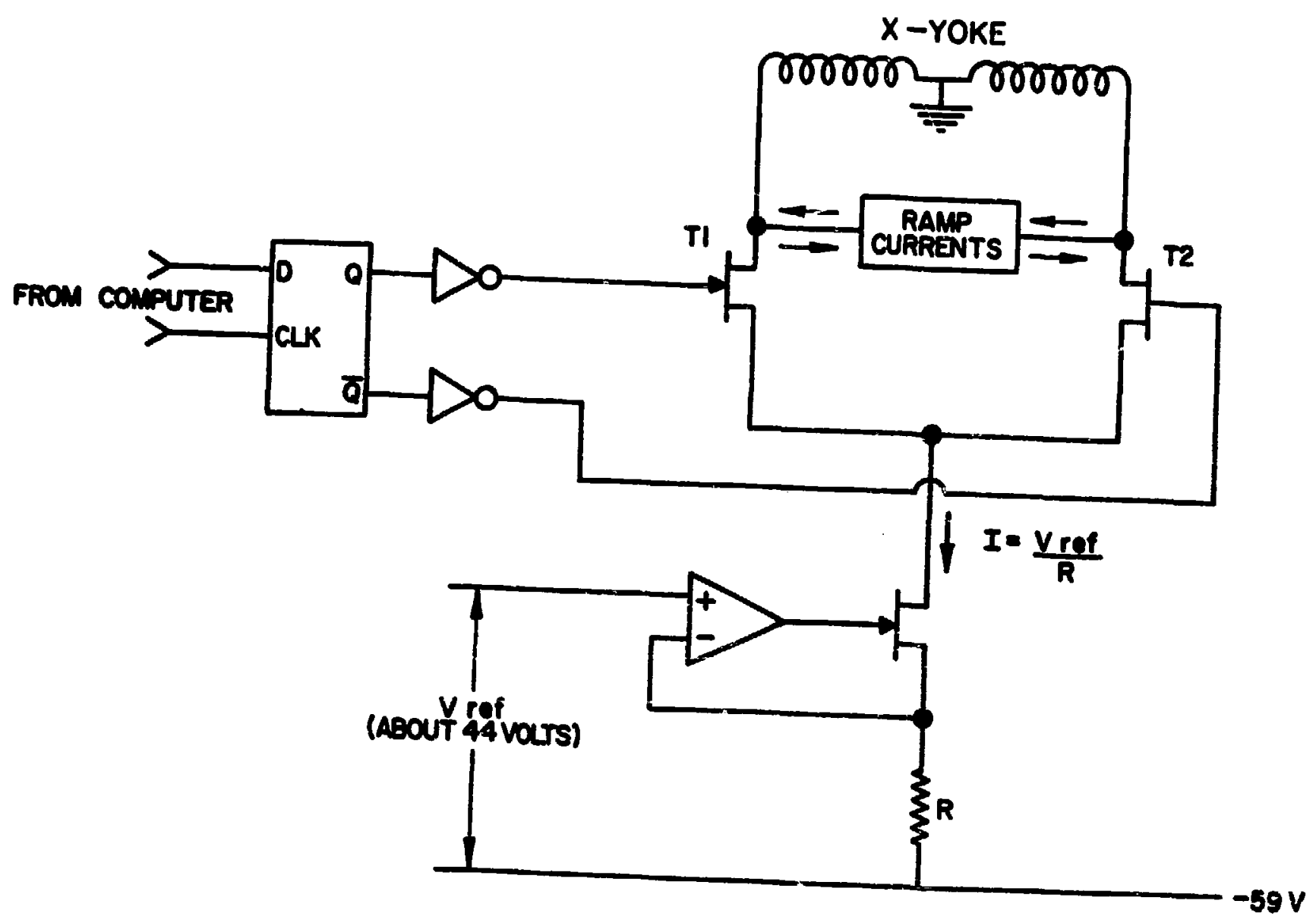

FIG.6 PRECISION D-A CONVERSION IONE DIGIT, FOR X ONLY: $Y$ IS IDENTICAL) 
III. Position Control of the Spot on the Precision CR T

\subsection{Precision Current Generators}

Figure 6 shows schematically how the current for a single binary digit is switched into the CRT yoke circuit for $X$. (The $Y$ circuit is identical). A reference voltage $V_{\text {ref }}$ produces a constant current $I$ in a precision resistor $R$. This current is diverted into one half of the balanced yoke or the other by switching the transistors $\mathrm{T} 1$ and $\mathrm{T} 2$. Important features of this circuit are that the resistor $R$ carries a constant current whethe $r$ the digit is in or out, avoiding thermal effects, and that the reference voltage source supplies no current. All power for the yoke is supplied from the $-59 \mathrm{~V}$ source, whose stability is not critical. The binary weighting of the yoke currents is determined by the value of the resistor $R$.

The reference voltage is conveniently used to add the pincushion distortion correction. The manner in which this is done will be described below. (Section 3. 3)

The current drivers for all digits are in parallel, and the currents are actually summed in the yoke. Also in parallel with the yoke are the drivers for the ramp circuits generating a slice line.

\subsection{Ramp Circuits}

Figure 7 shows how, on receipt of a signal from the computer, a ramp signal is generated for $X$ and another for $Y$. An attenuation factor of $n / 8(n=0,1,2 \ldots 7$, 8) is applied to $X$ and another to $Y$ to obtain the requisite scan orientation, and the polarity of $X$ or $Y$ is reversed, should the orientation require it. The ramp voltage so obtained is used to control a current driver for adding a ramp current to the appropriate $\mathrm{X}$ or $\mathrm{Y}$ yoke, and the voltage is used directly as input to the computing circuits for pincushion correction.

\section{3 Pincushion Correction Circuits}

For pincushion correction, the transformations

$$
\begin{aligned}
& X^{\prime}=X+a X\left(X^{2}+Y^{2}\right) \\
& Y^{\prime}=Y+a Y\left(X^{2}+Y^{2}\right)
\end{aligned}
$$




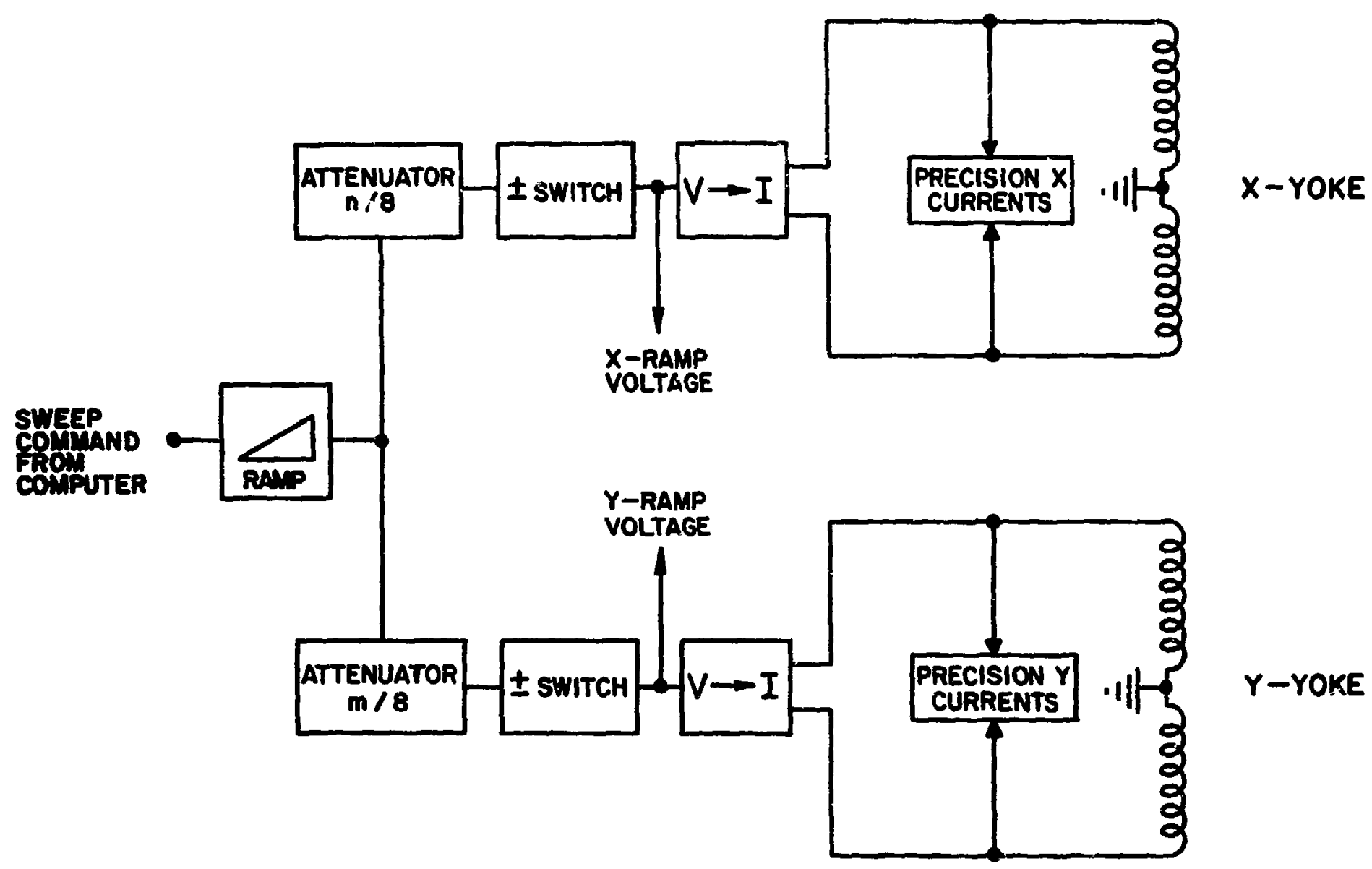

FIG.7 RAMP CIRCUITS 


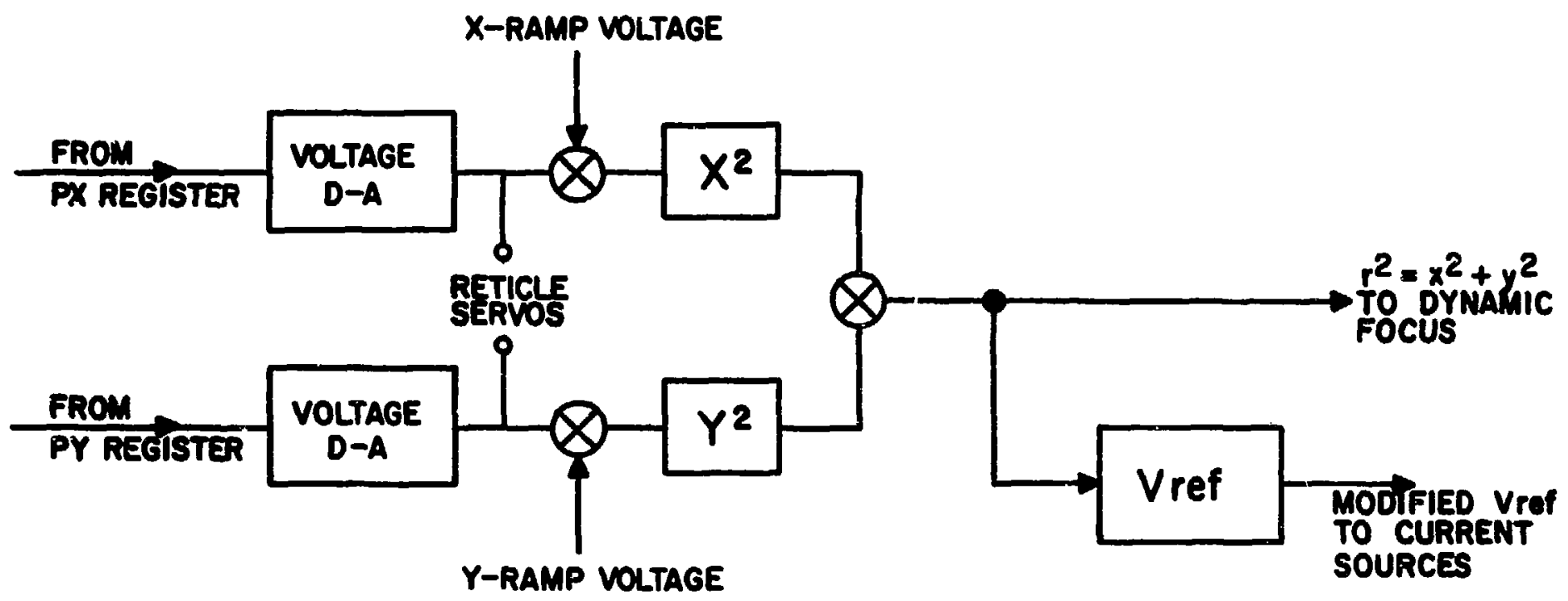

FIG. 8 PINCUSHION CORRECTION 
must be applied to the respective deflection currents. By using as input var1ables to this analog computation the correctly summed voltages arising from major deflection and ramp value, a voltage is obtained which is proportional to $\mathrm{X}^{2}+\mathrm{Y}^{2}$ and which can be made to adjust the yoke current appropriately by suding it to the reference voltage on which the yoke currents are based. Figure 8 shows how this is done. Note that the one $V$ ref is used as the $s$ tandard for ill digit values in $X$ and $Y$. 
IV. Specifications of some C ritical Components

4.1 Precision Lens

Magnification:

Illuminant:

Object size:

Image size:

Object-Image distance:

Relative Aperture:

Distortion:

Transmission:

Relative Illumination:

Resolution:
2:1 reduction

P-16 phosphor

6" $(15 \mathrm{~cm})$ diameter

$3 "(7.5 \mathrm{~cm})$ diameter

$32 \pm 0.2511(81.3 \pm .65 \mathrm{~cm})$

f 3.0 (at infinity); at 2:1 this corresponde to a

N. A. of $1 / 18$ on the object side

$0.1 \%$ Maximum

70\% Minimum

$70 \%$ of on-axis value at edge of field

$300 \mathrm{lp} / \mathrm{mm}$ or MTF response of $75 \%$ at

$60 \mathrm{lp} / \mathrm{mm}$

The lens was designed and made by OPTEK, Inc., Costa Mesa, California, and is their design number 1093.

\subsection{Deflection Yoke for Precisıon CRT}

Push-Pull Windings

Half-axis values:

Inductance

Resistance

Current

Residual Magnetism
$250 \mathrm{HH}$

$1 \Omega$ maximum

$2.1 \mathrm{~A} / 21^{\circ}$ deflection at $20 \mathrm{kV}$

$0.005 \%$ maximum

The yoke is made by CELCO and is their type HAD428-P560-105.

\subsection{Precision CR T}

Ferranti type $9 B 7100$

Gun Type: Tetrode, double cross-over. Type 71

Phosphor: Q-type (similar to $P-16$ )

Spot size: approximately 18 microns 
4. 4 Focus Coil

Ferranti type FC -7A

Contains: Static focus

Dynamic focus

Astigmatism Correction

\section{5 Centering Coil}

Ferranti FC-10A 


\section{The Discriminator Circuit}

The discriminator circuit has had to be completely re-designed for use with the low contrast Scotchlite film from the $12 \mathrm{ft}$. bubble chamber. (Incidentally, it even works well with conventional film. / By dispensing with the reference photomultiplier signal, and using auto-correlation instead of correlation, a considerable improvement in discrimination has been obtained.

Briefly, the average height of the pedestal on which the signal stood for the previous line is used as the reference level for the next one.

Figure 9 explains the general scheme. After passing through an active filter with a response similar to that of the usual sine-squared filter, the signal is attenuated by an amount proportional to the length of scan-line currently being used. (This is the slice-scan width W.) This is done so that time integration will yield the average height. The integrator is driven via a followand-hold circuit which can be held at its current value whenever a signal pulse is deiected. Thus during the time when a signal is present we go on integrating, but we only integrate the barkground level.

The average background height so obtained is sampled at the end of a sranline and held for use as a reference level during the passage of the next scanline. This is done by resetting, connecting and disconnerting the sampleand-hold circuit with a digital signal obtained from the POLLY logic.

The output of the sample-and-hoid circuit now passes through a DC amplifier whose gain is under computer control from the so-calied "Discriminator level" register. The task of the computer is to choose some amplification factor which will cause signal to be separated from background when the comparison is made. 


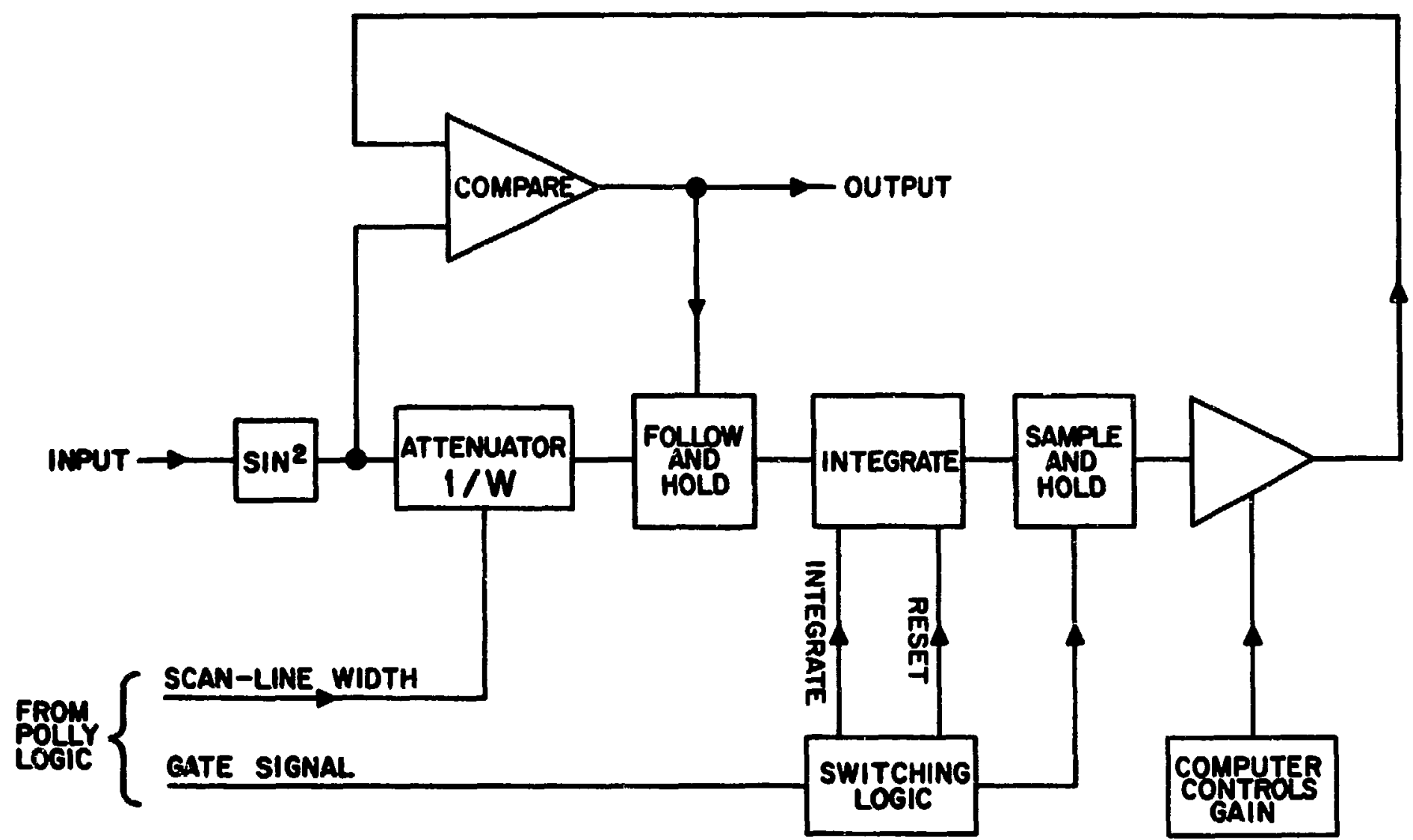




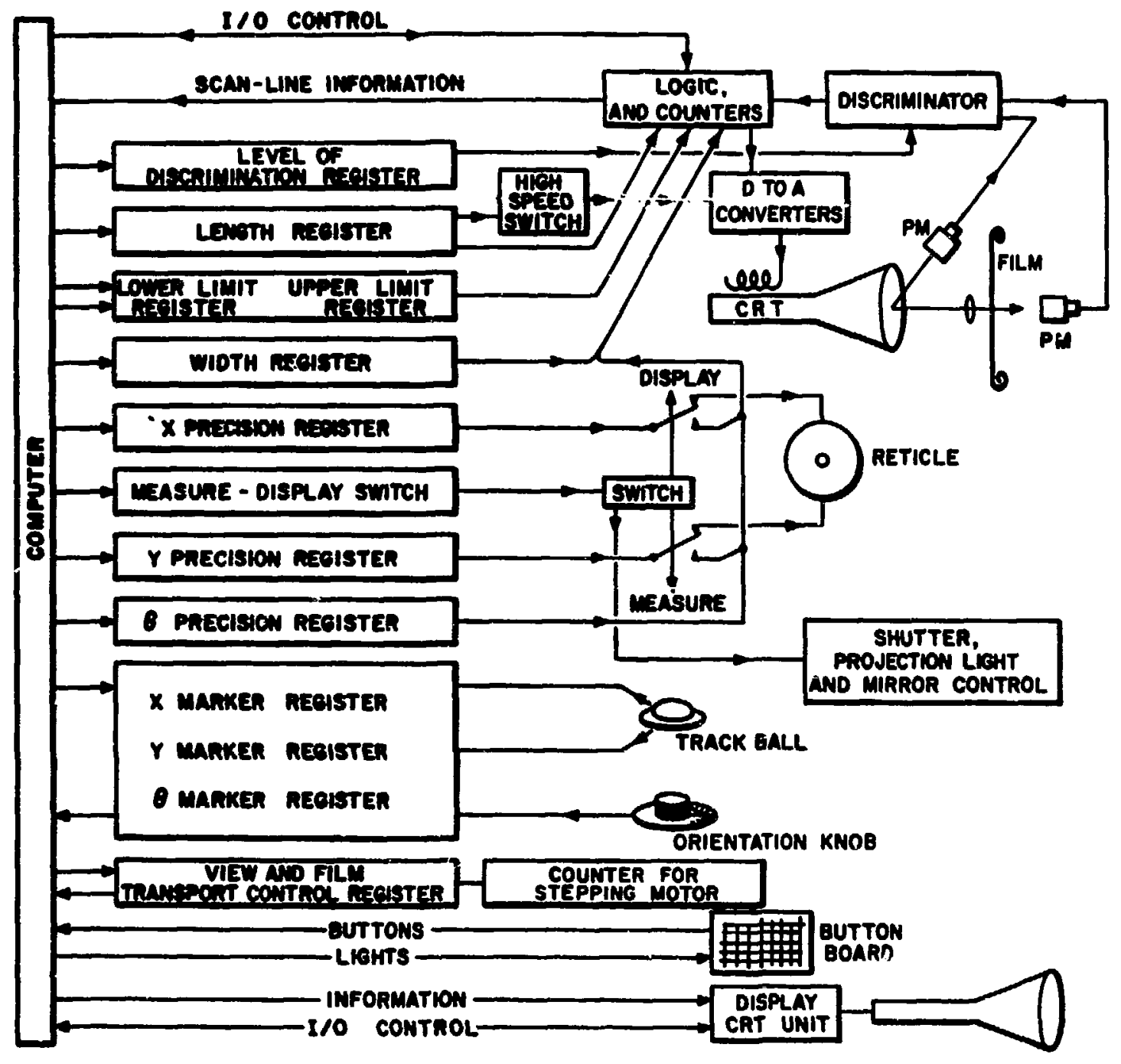

FIG. 10 POLLY III - USE OF REGISTERS AND MAIN INFORMATION FLOW 
VI. Description of the POLLY III Digit: Logic

\section{1 General}

POLLY can be switched into two modes, the display mode and the measuring mode. In the display mode, the operator is shown a direct projection of the film to be measured on which is superimposed a reticle whose coordinates are under computer control. In the measuring mode, the computer can instruct POLLY to scan a small section of the picture in a series of evenly spaced parallel lines of equal length and arbitrary direction. A number of parameters of this scan can be set by loading registers from the computer before the performance of the scan is requested. Figure 10 shows how the computer and all the registers are interconnected.

In both modes, the operator can increment or decrement three registers readable from the computer by using a track-ball for two registers called $\underline{X}$ and $Y$ and a knob for one called Theta. Also unaffected by the mode is a display unit on which pictures and writing may be drawn by the computer. This display unit can be made to regenerate its pirsure continuously from the computer memory without attention from the central proressor. A set of 32 buttons on the console of POLLY enables the operator to convey derision information to the computer.

The measuring mode can be selerted in two forms, the high-speed mode and the low-speed mode. Operation in the low-speed mode requires that three extra registers be set up to define the expected width of a track on the picture and the number of scan lines. This enabieg the machire to return extra information at the end of the scan process ;The high sped mode is selected by specifying zero scan-lines in one of the registers. :

\section{2 Digital Control of the Spot on the Precision CRT}

The spot, which is about $18 \mathrm{f}$ in diameter on the tube at haif-brightness can be deflected to any point on a grid with a mesh of 2 F. There are $32768 \times$ 32768 possible position. to which the spot can be moved, and every eighth one of these, i. e. $4096 \times 4096$, can be chosen by loading the $X$-precision and $Y$ precision registers. 
Based on the point so chosen, the spot is now deflected, under analog control, in a series of lines perpendicular to a base line whose angle is chosen by referring to the $\theta$-precision register. There are 64 directions, each corresponding to an angle of arctan $n / 8(n=1$ to 8 by integers) from a vertical or horizontal direction.

In the low speed mode, the spot is deflected at $4 \mu / \mu \mathrm{s}$ in one direction, while a clock counts microseconds. Simultaneously, the spot is deflected by a specific fraction $(8 / 8,7 / 8,6 / 8 \ldots 1 / 8,0 / 8)$ of this speed in the perpendicular direction. The clock is used also to reduce the value of the counter whose contents are copied initially from the width-precision register, (maximum initial value $=127$ ), and whose reduction to zero marks the end of a scan line.

\section{3 Information Retrieved from the Film}

The measurement of the width of a track or other marking on the film is accomplished by focusing an image of the spot on the film and measuring the light after its passage through the film by means of a photomultiplier and a specially controlled discriminator circuit described in Section $\mathrm{V}$. The clock is used to drive a counter which tallies $1 \mu \mathrm{s}$ intervals $(4 \mu$ projected lengthe) until a track-signal is encountered.

At this instant, the contents of the counter are copied into a "track edge" register and a second counter is s:arted. The second counter tallies $1 \mu \mathrm{s}$ intervals until the track-signal ceases when its contents are compared with the minimum and maximum permitted trark width in the limits register. If the signal was too thin, the process continues as thou $\mathrm{g}$ h the signal had never been seen. If the track was too thick, the value of the second counter is copied into a "track width" regiater and a "double-hit" indication is flagged. If the track has an acceptable width, the conteris of the second counter are copied into the "track width" register, and the search goes on. If any other - ignal, wider than the minimum occurs, the double-hit indicator is flagged. The canning of a line is terminated either by exhaustion of the width control or by the flagging of the double-hit indicator.

Simultaneously, the sigral comparison with the limits register is used for 
another purpose: If the width is between the limits, the line is designated a good hit; if the width is outside the limits, or if a second significant gignal has been detected on this scan line, the line is designated a bad hit. At this point five registers, initially zeroed when the entire scan was started, are updated. These registers are:

1. Sum of edge-counts for good lines (incremented with contents of first clock-driven counter if hit is good).

2. Sum of width-counts for good lines (incremented with contents of second clock-driven counter if hit is good).

3. Sum of line sequence numbers for good lines (incremerited with line sequence number if hit is good).

4. Number of good lines (incremented by 1 if hit is good).

5. Number of bad lines (incremented by 1 if hit is badj.

These five quantities are output to the computer following the last of the twobyte groups which emerge after every scan line. The number of scan lines requested can be controlled by the computer, but to inform the POLLY hardware of when to output these five sums there is a length register, whose value is in a counter, and is decremented by 1 for each scan line traversed. When this counter reaches zero, the five sums are sent to the computer. Each of the first three sums occupies a four-byte word. The fourth and fifth sums are in the third and fourth byte of a final word. If the length register is lcaded with a zero prior to a computer request for a slice-scian, the POLLY hardware will execute subsequent slice-scans in a high-speed mode: in this mode the projected speed of the spot is $16 \mu / \mu \mathrm{s}$. The speed of the clock is unchanged. Also the five sums are not output.

Each scan line thus gives three pieces of information: the edge-count from the beginning of the line to the first detection of the track, the width-count from first detection to last detection of the track, and a single yes-no indication if the discriminator sees any other acceptably wide track-signal between last detection and the exhaustion of the width-counter. These three items are packed into two, eight-bit bytes for output to the computer. When a line has been drawn on the precision CRT, the counters are eeroed 
and the spot is moved to its stirting position for the next line. This is repeated until the computer has received the correct number of bytes of information. The starting point of a new line is always a $2 \mu$ mesh-point. It is eight positions $(16 \mu)$ away in one direction and its displacement in the other direction is such that the line connecting starting points is perpendicular to the scan lines themselves. The starting coordinates are held in a pair of registers which are updated before the start of each new line. 


\section{The Indexing Motor}

Figure 11 shows the motor which is used for indexing the four-way mirror. A circle of solenoids is energized sequentially, causing the armature to rock on the ball-joint, the axis of rotation being a diameter of the armature.

Since the inner beveled gears have unequal numbers of teeth $n$ and $n+1$, and each of the in solenoids is equally spaced round the circle, the shaft gear and hence the shaft will be caused to rotate by an amount $2 \pi / \mathrm{mn}$ at each step. The armature is prevented from rotating (it nutates only) by the outer gears, which have equal numbers of teeth and supply no torque.

The wear on the beveled gears is even and equal, and should not affect the repeatability of the device.

The indexing motor is made in Salem, Massachusetts by the Measur-matic Corp., and is the subject of a U.S. patent. 


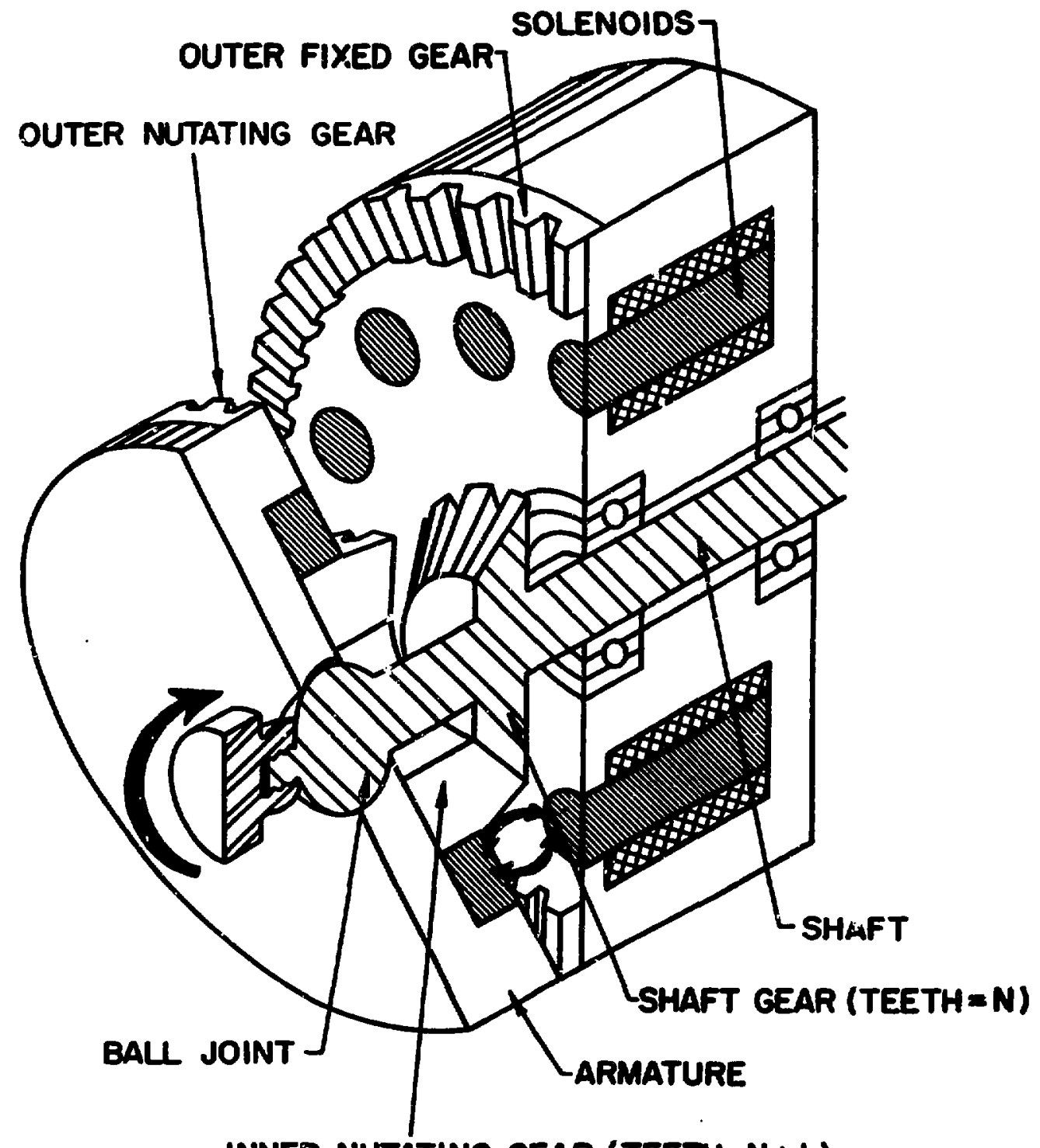

INNER NUTATING GEAR (TEETH $N+1$ )

FIG.II INDEXING MOTOR 
ViII. References

(1) 1. Pless, "The PEPR Syetem," IEEE Trans. Nucl. Sci., Vol. NS-12 279-290 (1965).

(2) R. K. Clark, et al., Methods of Computational Physice 5, 47-99, B. Alder, Editor Academic Preas, New York (1966).

(3) D. Hodges, et al., "POLLY: A High Precision Film Scauning and Measuring Syetem, " ANL Technical Memorasdum No. 91 (1964).

(4) W. A. Cooper, et al., Phys. Rev. Letters 20, 1059 (1968).

(5) W. A. Cooper, ot al., Nucl. Phyeice B16, 155-160 (1970).

(o) W. W. M. Allison, et al., Phys. Rev. Letters 24, 618 (1970).

(7) R. Davis, B. Werner, et ai., Nucl. Physice B23, 37-44 (1970).

(8) Y. Cho, M. Derrick, et al., Phys. Letters 32B, 409-412 (1970).

(9) Y. Cho, M. Derrick, et al., Phys. Rev. D37, 1561-1568 (1971).

(10) R. Barr, R. Claxk, D. Hodges, J. Loken, W. Manner, B. Musgrave, P. Pennock, R. Royeton and R. Wohman, Rev. Sci. Inst. 39, 1556 (1968).

(11) W. W. M. Allieun, F. Beck, D. Hodges, J. G. Loken, B. Musgrave, H. B. Phillips, R. Royston, R. A. Sunde, Nucl. Inet. and Methode 79, 341-345 (1970).

(12) ANL/HEP unpublished FOLLY Notes (see Appendix I).

(13) ANL/HEP 6916 - POLLY II: A Complete Syatem Incorporating Facilities for Meaeuring Bubble Chamber Film without Pre-Scanning;

Vol. 1: General Deecription and Program Manual

Vol. II: Program Text .

(14) F. Beck, "The Special Features of POLLY: How They are Used for Automatic Scanning, " IEEE Trans. on Computere (1971). 
IX. APPENIIX: POLLY Notes published to Jul;, 1971

POLI.Y Report No's., Subject, Author(o), and Date of each report

1 Plan for an Automatic Bubble Chamber Film Measuring System:

R. Royston, D. Hodges - January, 1967

2 External Device Control of POLLY I (PDP-7 Computer):

D. Hodges, P. Wehman, G. Wittmus - March, 1967

3 POLLY Syetem for Bubble Chamber Film Measuring:

D. Hodges - January, 1967

4 Use of Interactive Graphical Consoles in Bubble Chamber Film Analyoiu:

R. K. Clark

5 First 12 Evente Measured on CHLOE - Preliminary Results:

R. Royatos - January, 1967

6 Circle Extrapolation:

J. G. Loken - May, 1967

7 Calibration of CHLOE:

P. R. Pennosk - April, 1967

8 External Device Contrul of POLLY Il (Sigma 7 Computer):

D. liodges, G. Wittrnus, - June, 1967

9 An Interactive System for Bubble Chamber Film Measurement:

R. Clark, D. Hodges, J. Loken, B. Muegrave, P. Pennock, B. Phillipe,

R. Royston, R. Wehman, and G. Wittmus

10 Sigma 7 Display Device - Programming:

D. Hodges, G. Wittmus - June, 1967

11 Operating Instructions for the PDP-7 Program to Calibrate POLLY I:

D. A. Henderson, P. Pennock - July, 1967

12 Accuracy of the POLLY Calibration Grid:

D. A. Henderson - July, 1967

13 Operating Inatructione for the PRODUCTION VII POLLY I Syetem:

D. Hodgen, J. Loken, B. Muagrave - September, 1967

11 A PDP -7 Triple Precioion Float Point Package:

D. A. Henderson - Auguet, 1967

15 Operating Proceduree for POLLY I Hardware:

R. Barr, D. Hodges, H. George, R. Sunde, R. Wehman - October, 1967 
16 Fiducial Mezturement of POLLY I:
B. Musgrave - November, 1967

17 Comparison of Fiducial Mark Measurements made with POLLY I, Hermes and

D. Rhines, B. Musgrave - January, 1968

Olivia:

18 Track Matching in Bubble Chamber Geometry Programs:

W. Manner - March, 1968

19 POLLY I Calibrate Measuring:

P. Pennock - June, 1968

20 POLLY I Calibrate Fitting:

P. Pennock - June, 1968

21 Scan Cards to DEC Tape:

P. Pennock - June, 1968

22 Deflection Defocusing in CRT's:

H. Phillips - July, 1968

23 CRT Deflection Errors:

H. Phillips

24 Analysis of Bubble Density Measurements on POLLY I:

D. Johnson, J. Loken, T. Wangler - January, 1969

25 Machine Coded Subrcutines in Mosam \& Calibrate: Instructions for their use F. Beck - January, 1969 Under the Batch Proceseing Monitor

26 Stucy of POLLY Events Trailing Geometrical Reconstruction:

D. Pollard - January, 1969

27 POLLY III: Preliminary Specification for a Four-Strip Measuring Machine for Film from $12 \mathrm{ft}$. and other Bubble Chambers:

F. Beck, H. Phillips - May, 1969

27a 1at change to the POLLY III Specification:

F. Beck - June, 1969

27b 2nd change to the POLLY II: Specification:

W. W. Allison, F. Beck, R. C. Zieman - July, 1969

28 Automatic Scanning and Measuring of POLLY II:

W. W. Allison, J. Loken - May, 1969

29 POLLY II Production:

R. Sunde - Septomber, 1969 
30 Operation of the POLLY III Logic:

F. Beck, R. Zieman - April, 1970

31 Pincushion Errors in the POLLY Slice-Scan:

H. O'Donnell, C. J. Robinson - May, 1970

32 Automatic Use of POLLY II Ionization Data for $2.3 \mathrm{GeV} / \mathrm{c} \overline{\mathrm{p}} \mathrm{p}$ experiment:

D. Rhines - Octoker, 1970

33 Notes on the Mechanical Design of POLLY III:

C. Lindenmeyer - January, 1971

34 Tests on the ROTO-DIGITIZER

J. Foreman - July, 1971 